\title{
Redundant Timing Crosschecking for Frontend Digitization Systems: Principle and Validation Tests
}

\author{
Jinyuan $\mathrm{Wu}$, Daren Chen and Jingjing $\mathrm{Xu}$
}

\begin{abstract}
In contemporary high energy physics experiments, in addition to the regular three dimensions, the fourth dimension, or the timing information of the charged particle hits becomes necessary. It would be very useful for the frontend digitization system to include a feature of redundant timing crosschecking. The inspiration for the timing crosschecking scheme came from the long-abandoned analog mean-timer schemes. In this mean time based scheme, a cable set with taps connected to the digitization modules is utilized as a pulse path and pulses are driven from both ends alternately without overlapping. The mean times of arrival times of the pulses in different modules represent a common time standard and their variations is recorded and monitored. The time delay drifts of the cable segments are self-cancelled in this scheme and this feature enables easy achievement of good timing precision without the need for complex hardware.
\end{abstract}

Index Terms - Time to Digital Convertor, Timing Reference Distribution, Mean Time

\section{INTRODUCTION}

$\mathrm{I}_{\mathrm{a}}^{\mathrm{N}}$ contemporary high energy physics experiments, in addition to the regular three dimensions, the fourth dimension, or the timing information of the charged particle hits becomes necessary. Timing information can be used for particle identification or vertex recognition in track reconstruction. With the progress of detector technology development, timing measurement precision better than $30 \mathrm{ps}$ becomes achievable.

Obviously, at the frontend electronics and digitization modules of the detector, a precise timing reference must established in order to make a useful timing measurement. In most cases, the timing reference at the digitization module is a stable and low jitter clock signal that is driven by a common crystal source.

The frontend and digitization modules are usually installed deep inside the detector and are connected to the control units

Manuscript received Oct. 26, 2019. This work was supported in part by Fermi Research Alliance, LLC under Contract No. DE-AC02-07CH11359 with the United States Department of Energy.

Jinyuan $\mathrm{Wu}$ is with Fermi National Accelerator Laboratory, Batavia, IL 60510 USA (phone: 630-840-8911; fax: 630-840-2950; e-mail: jywu168@ fnal.gov). Darren Chen is with Hinsdale Central High School, 5500 South Grant Street, Hinsdale, IL 60521, USA (email: 819008@hinsdale86.org). Jingjing $\mathrm{Xu}$ is with Purdue University, 610 Purdue Mall, West Lafayette, IN 47907, USA.

978-1-7281-2322-6/19/\$31.00 C2019 IEEE in the control room through data link, typically with optical fibers. It is a natural choice to use the downward link to transfer data back while use the upward link to send the common system clock along with a small amount of control commands.

The regular vertical paths of clock and data link might be about 100 meters in large model detectors so temperature effect must be taken into account. While there exist various solutions in the vertical clock distribution paths, it would be very useful to build a horizontal connection to provide a redundant timing crosschecking as shown in Fig. 1.

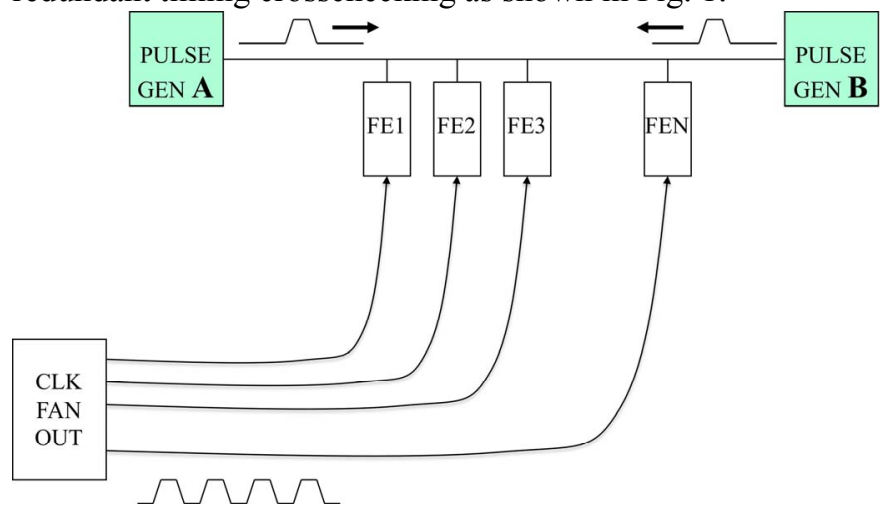

Fig. 1. A possible timing crosschecking scheme

The inspiration for the timing crosschecking scheme came from the long-abandoned analog mean-timer schemes [1-4]. Some brief study of the authors can be found in References [5] and [6]. Consider a detector subsystem containing $\mathrm{N}$ frontend electronics and digitization modules, marked as FE1, FE2, etc. Each module is connected to the clock fanout units so that a common clock signal is distributed to each module. In addition to the regular vertical path, the modules are interconnected through a cable with pulse generators sending pulses alternately without overlapping from both ends. The pulse generators are designed with internal impedance matching the cable impedance to eliminate signal reflection and each frontend module picks up the pulse voltage with a high impedance buffer. 
The arrival times of the pulses from both directions are digitized in each digitization module and these times are used to check and monitor the system timing reference in the frontend digitization modules established via the vertical paths.

Clearly there are propagation delays between modules when the pulses travel in the cable and the propagation delays may change as temperature changes. Also the timing jitters of the pulse generators may not be small. So the arrival time of an individual pulse digitized in a module is not a good timing standard. But it can be shown that the mean time of the arrival times of the pulses from both directions is the same in all modules which can be used as a common timing standard for system timing crosschecking. Note that this common timing standard is established by using mathematical cancellation instead of high performance devices. Variations due to temperature changes are eliminated automatically in this scheme.

In this paper, we will discuss the principal of this scheme in Section II. Preliminary test results will be described in the remaining sections.

\section{Property OF THE MeAn Times}

Consider two pulses traveling in opposite directions alternately without overlapping. The arrival times of the two pulses are measured by any two modules $\mathrm{FE}_{\mathrm{i}}$ and $\mathrm{FE}_{\mathrm{j}}$ as shown in Fig. 2.

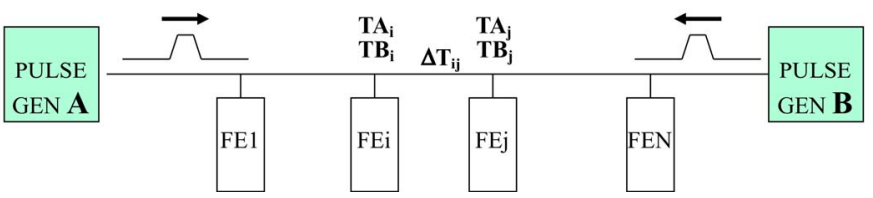

Fig. 2. The arrival times of the pulses

For simplicity, let's define the arrival times for only one edge, for example, the leading edges of the pulses, although the following calculation applies to either edge. If the arrival time of pulse $\mathrm{A}$ at module $\mathrm{FE}_{\mathrm{i}}$ is $\mathrm{TA}_{\mathrm{i}}$, then the arrival time at $\mathrm{FE}_{\mathrm{j}}$ is $\mathrm{TA}_{\mathrm{j}}$ which is later than $\mathrm{TA}_{\mathrm{i}}$.

$=+\Delta$

The propagation delay of the pulses in the cable segment between two modules is denoted as $\Delta \mathrm{T}_{\mathrm{ij}}$. The arrival times for pulse $\mathrm{B}$ have similar relation.

$=+\Delta$

Here, we assumed that the propagation delays for pulses A and B traveling in opposite directions are the same, which is approximately true for most cables with acceptable quality.

The mean times of the arrival times of the two pulses at two modules can be simply calculated.

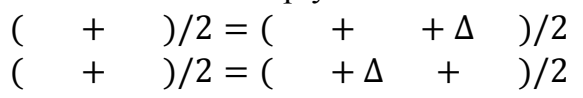

It can be seen that these two values are identical as long as the propagation delays for the pulses traveling in both directions are the same. If the temperature variation causes the propagation delay to change, the mean times measured and calculated at both modules will change, but by the same amount. Similarly, if the pulses from pulse generators are launched earlier or later, the values of the mean times will change but remain equal to each other.

This property of the mean times is true for all modules in the same cable. As pointed out early, the arrival times are not limited to the leading edges of the pulses. Also, there is no restriction on how many pulses can be sent out from each side. For example, pulse generator A can send out a burst of pulses and then $\mathrm{B}$ can send out a burst with same number of transitions. This "common burst" scheme produces more time measurements in unit time period that helps to improve measurement precision.

In practical implementation, the two pulse generators can be two driving channels of a single module to reduce complexity of the system. In fact, a separate pulse generator can be totally eliminated and the driving channels can be implemented in the frontend modules. During actual operation, only the driving channels of the end modules in each cable chain are enabled.

It is preferable to use differential signaling standards such as LVDS for the pulse link, but single ended coaxial pulses are able to achieve an acceptable performance, as in our evaluation test.

\section{The Evaluation Test Results}

The evaluation tests of the timing crosschecking scheme are performed in a digital oscilloscope (Tektronix DPO7254C) with sampling rate of 10 GSPS. An arbitrary function generator (Tektronix AFG3252) is used to send pulses alternately into a cable chain from both ends. The cable set consists five $50 \mathrm{Ohm}$ coaxial cable segments, joined together with $4 \mathrm{BNC}$ tee connectors. Each tee connector is plugged into a oscilloscope channel with high impedance setting and the testing waveforms are shown in Fig. 3. 


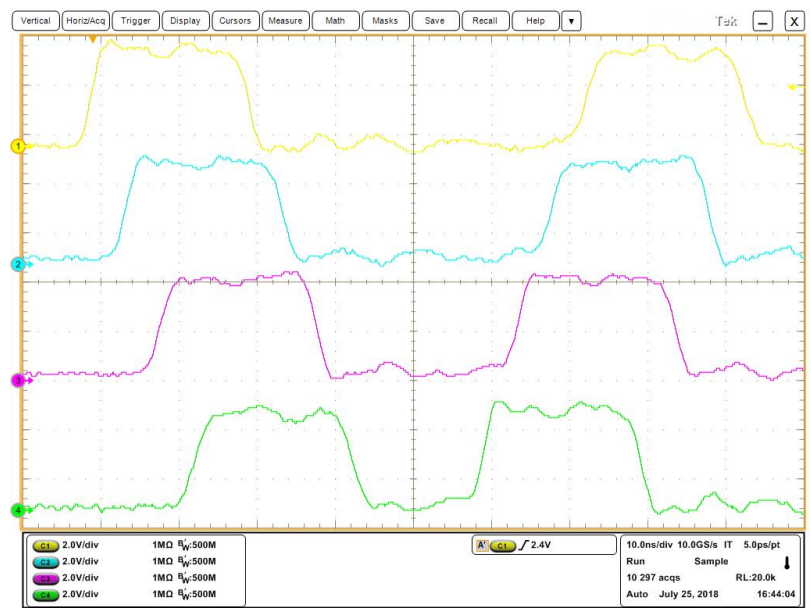

Fig. 3. The test waveforms

Each oscilloscope channel emulates a frontend digitization module and lengths of the cable segment delays between channels are approximately 4 ns each. The first pulse shown is driven out from channel $\mathrm{A}$, which propagates in the direction from oscilloscope channel 1 to channel 4 and the second pulse is from channel B which travels in the reversed direction.

The waveform data are stored in text files and are readout for analysis. A voltage threshold $(1.25 \mathrm{~V}$ typical) is defined to extract the edge transition time and the time of passing threshold is rounded to the nearest $100 \mathrm{ps}$ which is the natural sampling period of the oscilloscope. We intentionally didn't use further analog data information so that the time extraction process behaves as closely as possible to a TDC device with a 100 ps LSB.

Four edge transition times for each channel, i.e., both leading and trailing edges for both pulse A and B are extracted and the mean of these four times is calculated. The edge transition times and the mean time for all channels are shown in Fig. 4.
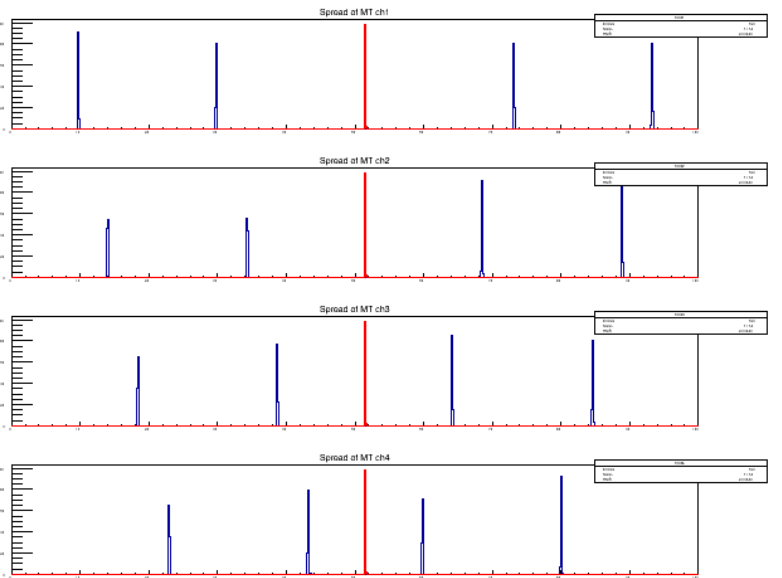

Fig. 4. The edge transition times (blue) and the mean time (red) for all channels

It can be seen from the histogram above that the mean times for all four channels are approximately the same, even though the arrival times of the pulse edges are significantly different due to cable propagation delays.

The differences of the mean times between any two channels are small constants and the variations reflect the clocking skews and jitters between oscilloscope channels. The standard deviations of the mean time differences are in the order of about $30 \mathrm{ps}$, which are essentially due to the quantization error of the $100 \mathrm{ps}$ timing measurement LSB. An example of the differences of the mean times are shown in Fig. 5.

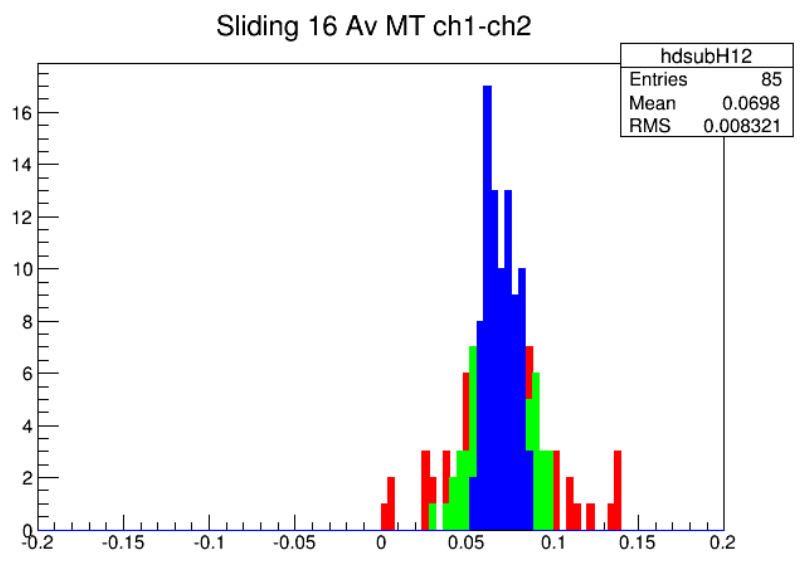

Fig. 5. The differences of the mean times 
The histogram plotted in red above reflects the raw differences of the mean times for channel 1 and 2 with standard deviation of about $30 \mathrm{ps}$. The green histogram is the distribution of the sliding average of 4 measurements. It can be seen that the width is clearly narrower due to averaging.

When more averages are utilized, the measurement precision is improved. The blue histogram reflects a sliding average of 16 measurements. In this case, the RMS timing measurement precision is better than $10 \mathrm{ps,}$, which is sufficient for timing crosschecking in most detector systems.

\section{The EFFects of CABle Delay Variations}

To study the effects of cable delay variations, we have adjusted some timing delays in the function generator, which equivalent to changing length of the pulse delivery cable segment. To exaggerate variations, delays of $1 \mathrm{~ns}$ and $5 \mathrm{~ns}$ are added and measurements are taken in all these conditions. The waveforms for these tests are shown in Fig. 6.

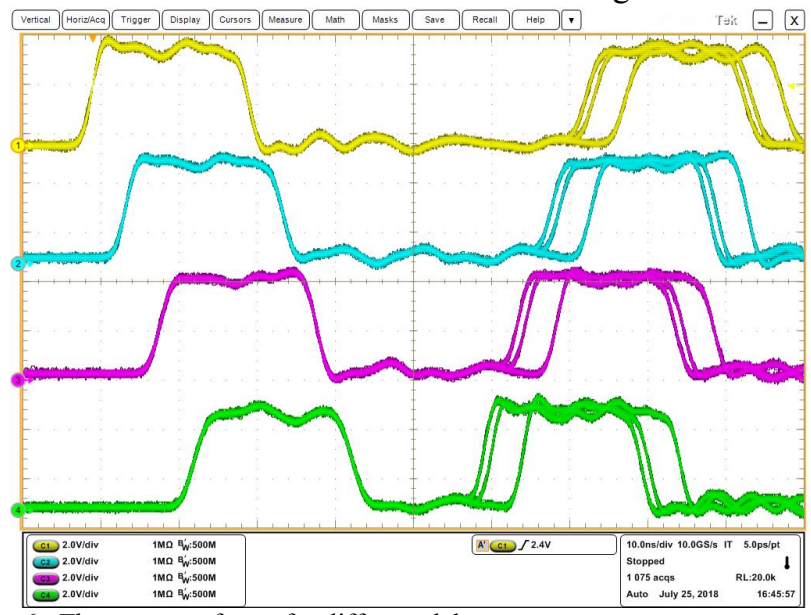

Fig. 6. The test waveforms for different delays

The edge transition times for all tests are extracted and the mean times for each channel in each case are calculated. As expected, the mean times in each channel will change as the delay changes. The edge transition times and the mean times for different delays are shown in Fig. 7.


Fig. 7. The edge transition times and the mean times for different delays

It can be seen that the mean times in different channels changes by the same amount for different delays. Therefore, the differences of the mean times between any two channels should remain the same even when the cable delay varies.

The histograms of differences of the mean times for the tests with different delays are plotted in Fig. 8. Note that data with all three delay settings are already blended together in these plots.

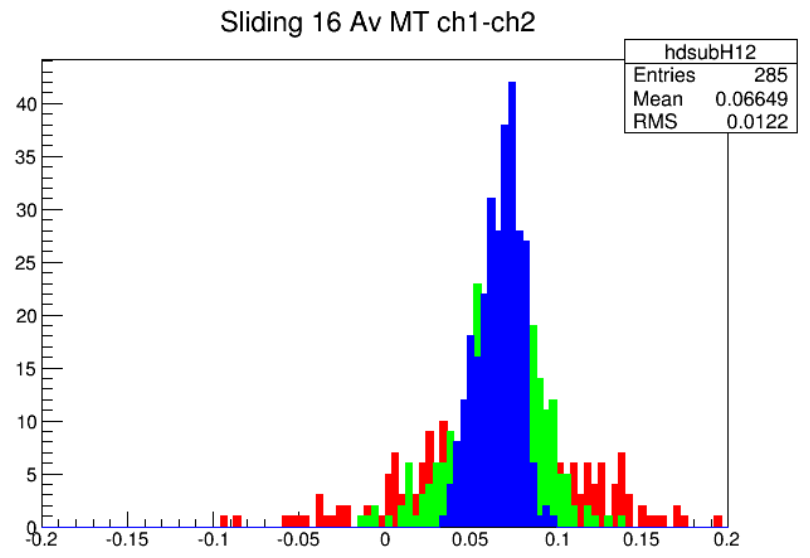

Fig. 8. The differences of the mean times for the tests with different delays

As we can see the mean times measured different digitization modules with the same timing standard when the cable delay changes. With a reasonable average, the timing crosschecking precision remains in the order of $10 \mathrm{ps}$. 
Comparing Fig. 5 and Fig 8, we do see that the distribution widths of the mean time differences become wider when the cable delay changes. This might be due to some higher order effects, such as shifts of the waveform DC baseline. Further study is needed to understand this better. But even with such exaggerated delay variations, an acceptable timing precision is still achievable.

\section{DISCUSSION AND FUTURE WORK}

The feature of the cable delay self-cancellation is the key to the effectiveness and simplicity of this mean time based timing crosschecking scheme. The results of the simple evaluation tests show that a satisfactory timing precision is achievable, even with moderate interconnection and far from optimal digitization hardware.

Since the timing precision can be improved with averaging of multiple measurements, it is unnecessary to push the TDC and related hardware to the maximum performance. Further study should be focused on simplicity and power consumption when the timing crosschecking function is included in the frontend digitization modules.

\section{REFERENCES}

[1] V. Sandberg, et al., "A Fast Analog Mean-Timer," in Nuclear Instruments and Methods in Physics Research, 1985, Pages 512 - 516, vol. A234.

[2] A. Cavestro, et al., "An Analog Mean-Timer for Long Scintillation Counters," in Nuclear Instruments and Methods in Physics Research, 1991, Pages 488 - 491, vol. A305.

[3] W. Grover, "A New Method for Clock Distribution," in IEEE Transactions on Circuits \& Systems, 1994, Pages 149 - 160, vol. 41.

[4] D. Rolston, et al., "A Distributed Synchronized Clocking Method," in IEEE Transactions on Circuits \& Systems, 2005, Pages 1597 - 1607, vol. 52.

[5] J. Wu, "An FPGA Wave Union TDC for Time-of-Flight Applications", in 2009 IEEE Nuclear Science Symposium Conference Record, 25-31 Oct. 2009 Page(s): 299-304

[6] H. Sadrozinski \& J. Wu, "Applications of Field-Programmable Gate Arrays in Scientific Research”, Taylor \& Francis, December 2010. 